\title{
Learning from your mistakes: is aneuploidy so bad, after all?
}

\author{
David F. Albertini
}

Published online: 17 March 2012

(C) Springer Science+Business Media, LLC 2012

It remains a hallmark of human reproduction - and a thorn in the side of so many older patients who have retained the right to reproduce later in life - that the gametes and embryos of Homo sapiens display a very high index of chromosome imbalance. Known most commonly as aneuploidy, the state cells find themselves in, when harboring a chromosome constitution that diverges from the euploid number of 46, this particular "abnormality" often assumes a causative attribution for the age-related decline in gamete quality. This not only decreases the chances of maintaining a term pregnancy but, for some chromosomes, will predispose offspring to a range of well known genetic diseases, such as Downs Syndrome. For students of reproductive medicine, this basic fact has been staring us in the face for more than 75 years now, and continues to stimulate research that aims at explaining the molecular underpinnings of this disturbing and enigmatic predisposition. We should bear in mind, further, that the incidence and age-related increase in aneuploidy is unique to humans, relative to other eutherian mammals studied to date.

In the general biomedical vernacular, cells and tissues that bear the "stigma" of aneuploidy are often cast in the dimension of frank cancer or are assumed to be well on their way to neoplastic or precancerous status. With the advent of new techniques and the emergence of the field of preimplantation genetic diagnosis (PGD), the karyotypic persona

Capsule The long held notion that aneuploidy is a bad thing for human gametes and embryos requires reconsideration in light of new studies on model organisms suggesting that there may be advantages to spending some time "imbalanced" when adapting to environmental stressors.

D. F. Albertini $(\bowtie)$

University of Kansas Medical Center,

Kansas City, KS 66103, USA

e-mail: dalbertini@kumc.edu of the human genome has been scrutinized and exposed, quantitatively and qualitatively, to an extremely high level of resolution. Coupled with the incredible growth and application of human ARTs worldwide, the chromosome constitution of human gametes and embryos has been systematically revealed in such detail as to advance materially our level of comprehension of the incidence and possible causes of aneuploidy - at least in the context of reproductive medicine. As a consequence then, our biases towards aneuploidy being a "bad thing" have been enhanced, our wariness of the aging problem has been reinforced, and now more than ever, the right to link genetic and epigenetic disturbances to human health and disease is more in evidence. Our issue this month fuels the fire ever more on the clinically pressing subject of age-related aneuploidy, ironically just as new research appears suggesting that aneuploidy may not deserve the bad rap it has shouldered historically, and may in fact be a good thing!

Besides the aging factor in females, and which is receiving more attention and support on the other side of the coin as a root cause of male infertility (see article from Zhu and colleagues, "Effect of male age on the outcome of in vitro fertilization: oocyte donation as a model"), many factors have been implicated in tipping the delicate balance between euploid and aneuploid, in the world of human ARTs. Ocal and colleagues report an association between follicular fluid levels of homocysteine and embryo quality ("The association between homocysteine in the follicular fluid with embryo quality and pregnancy rate in assisted reproductive techniques")and Combelles et al., now report a positive relationship between embryo quality and the release of superoxide dismutase 1 (SOD1) from in vitro-produced human embryos. That dietary and lifestyle factors contribute to aneuploidying-promoting oxidative stress conditions is also suggested by the studies of Eryilmaz et al., who introduce a role for vitamin E supplementation in the fight 
against endogenous or exogenous sources of reactive oxidative species (ROS). Unfortunately, these papers fail to provide a tractable link between aneuploidy and ageassociated changes in metabolism.

More to the point is the fact that aneuploidy happens, and at a rate that suggests the human conceptus and its gametic forbears are either prone to chromosome missegregation or do this intentionally, for some strange reason. Could gametes and/or blastomeres be taking high-stake risks by reshuffling the deck again, well after homologous recombination of meiotic prophase? And why would they do this when passing an aneuploid condition to trophectodermal or inner cell mass lineages of the embryo would guarantee the death sentence? Maybe such a fate is not the case. Many PGD studies have confirmed the fact that grossly aneuploid or mixoploid embryos, diagnosed at early cleavage stages, will somehow "right the ship" and end up producing completely euploid embryos that go on to produce genetically healthy offspring. This is a little bit like a magician pulling a rabbit out of a hata miraculous recovery from a soured aneuploid condition followed by magically corrective measures re-establishing euploidy through and beyond a term pregnancy.

Remarkable as they seem, these observations beg the question as to whether there is value-added or an adaptive advantage conferred upon embryos treading precariously, and in a chromosomally imbalanced state, in the interval between fertilization and implantation?

Now enter the provocative findings from the laboratory of Rong $\mathrm{Li}$ at the Stowers Institute for Medical Research, showing in a recent Nature article (doi:10.1038/nature10795) that the notion that cells exhibiting chromosome instability (CIN) are doomed to a genetic cul de sac may not be correct after all. Rather, it seems that under stressful conditions, yeast cells use aneuploidy as a form of megamutation that will lead them back to a successfully breeding euploid population, once they have culled out a genetic advantage for dealing with environmental stressors of various kinds. It is noteworthy that the kinds of stressors imposed on the yeast cells - oxidative, ER, and translational varieties - are not dissimilar from the stressors human gametes and embryos encounter on their way to the uterus or nitrogen dewar in the daily practice of human
ARTs. We must, however, bear in mind that this work comes from the study of yeast, an organism that gave the field of cell cycle control an enormous boost by virtue of its genetic tractability, and ushered in the concept of cell cycle checkpoints, and one bearing little resemblance to human gametes or embryos, when it comes to reproductive strategy. Better then, to ask whether evidence exists that might support the notion of a beneficial effect for aneuploidy on the road to and through human embryogenesis.

As a point of departure, consider first that the mechanistic causes of aneuploidy in human gametes and embryos have been thought to reside in defects in the construction of the spindle and/or in the vital attachments that chromosomes make with microtubules via kinetochores. Interestingly, the yeast experiments not only pinpoint the propensity to go aneuploid on the heat shock protein 90 (HSP90) gene product but also on the role this protein plays in chromosome segregation and kinetochore assembly, two signature events prone to dysfunction in the human oocyte and embryo. And in the matter of checkpoint control, the Nature study further suggests that failed assembly of the kinetochore could negate implementation of the spindle assembly checkpoint, creating a situation that would foster chromosome missegregation. This idea is consistent with work published in JARG demonstrating that checkpoint control gene products are deficient in the human embryo. Finally, and to the point of switching from aneuploid to euploid, as we have already considered above, several studies now indicate that human stem cells derived from blastocysts exhibit both the ability to propagate "stable" aneuploid and polyploid lines and in a recent paper in JARG, such stem cell lines were reported to revert to a diploid state, after further passaging!

While there is a long way to go before we can more fully understand the complexity of human gamete and embryo genetic integrity, the ever present problem of aneuploidy may be taking an unexpected turn that will keep clinicians and basic scientists alike both baffled and bemused into the near future. This may be one example of why carrying our biases into the discourse on how to make a good embryo will need to be turned upside down to gain perspective into what is normal. The message: keep your options open — like a good embryo! 